\title{
Millisecond Pulsars Modify the Radio-SFR Correlation
}

\author{
Takahiro Sudoh, ${ }^{a, *} \operatorname{Tim}_{\text {Linden }}^{b}$ and John F. Beacom ${ }^{a, c, d}$
}

${ }^{a}$ Center for Cosmology and AstroParticle Physics (CCAPP), Ohio State University, Columbus, OH 43210, USA

${ }^{b}$ Stockholm University and the Oskar Klein Centre, Stockholm, 10691, Sweden

${ }^{c}$ Department of Physics, Ohio State University, Columbus, OH 43210, USA

${ }^{d}$ Department of Astronomy, Ohio State University, Columbus, OH 43210, USA

E-mail: sudoh.611@gmail.com, linden@fysik.su.se, beacom.7@osu.edu

The observed correlation between the far-infrared and radio luminosities of galaxies illustrates the close connection between star formation and cosmic-ray production. Intriguingly, recent LOFAR observations find a peculiar radio excess in galaxies with low star-formation rates and high stellar masses. We show that recycled/millisecond pulsars (MSPs) can dominate the nonthermal emission in these massive quiescent galaxies and explain the excess. This is in line with recent gamma-ray observation suggesting that MSPs may efficiently accelerate cosmic-ray electrons. We find that MSP-based models provide a significantly improved fit to the LOFAR data. We discuss implications for the radio and gamma-ray excesses in M31 and local electron and positron observations.

$37^{\text {th }}$ International Cosmic Ray Conference (ICRC 2021)

July 12 th - 23rd, 2021

Online - Berlin, Germany

\footnotetext{
*Presenter
} 


\section{Introduction}

The correlation between galactic radio luminosities and star-formation rates (SFRs) has been intensively studied over many years (see, e.g., [1]). Observations have revealed a close correlation between these two variables, illuminating the link between cosmic-ray (CR) production and star formation. Gürkan et al. (Ref.[2], hereafter G18) examined this correlation at low frequencies $(150 \mathrm{MHz})$ with LOFAR observations of nearby galaxies. The bulk of their samples, which have modest or high SFRs ( $\gtrsim 1 \mathrm{M}_{\odot} \mathrm{yr}^{-1}$ ), are in line with the expectations that the radio luminosities are tightly correlated with SFRs. However, G18 also finds excess radio emission in galaxies with low SFR and high mass. There has been no clear explanation for this observation.

Here, we propose that millisecond pulsars (MSPs) may significantly contribute to the radio luminosities of low-SFR and high-mass galaxies, explaining this observation. This class of source has been paid little attention to in previous studies of galactic radio emission. However, an increasing number of observations suggest that MSPs may efficiently produce CR electrons and positrons (hereafter, electrons). These electrons produce diffuse non-thermal radio emission due to the synchrotron emission in galactic magnetic fields. Unlike supernova remnants (SNRs) and normal pulsars, which are commonly thought to be the sources of CR electrons in galaxies, the energetics of MSPs are not correlated with the prompt SFR for two reasons. First, MSPs are produced by the "recycling" of old pulsars, and they are typically formed $\sim$ Gyr after star formation [3]. Second, MSPs have a long lifetime, possibly reaching close to the age of the Universe [4, 5]. Therefore, MSPs can be an important source of CR electrons in galaxies that currently are not forming stars. Indeed, MSPs have been discussed in the context of $\gamma$-ray emission from globular clusters and Galactic bulge.

We argue that MSPs can be dominant over other sources of CR electrons in low-SFR and highmass galaxies. We then analyze the LOFAR data used in G18, finding that our MSP-based model significantly improves the fit. Our results have broad implications for galactic and CR physics. Details of our methodology and assumptions are given in our published paper [6].

\section{Theoretical Models for Nonthermal Radio Emission}

The dominant sources of CR electrons in star-forming galaxies (SFGs) are usually thought to be the shock wave produced by SNRs. The typical energy fraction transferred from SNR kinetic energy to CR electrons, $\eta_{e}^{\mathrm{SN}}$, is thought to be $\sim 10^{-3}$. Assuming a core-collapse supernova rate of $0.015 \psi \mathrm{yr}^{-1}$, where $\psi$ is the galactic SFR in $M_{\odot} \mathrm{yr}^{-1}$, we obtain a steady-state electron injection power from SNRs:

$$
Q_{e}^{\mathrm{SN}, \text { prim. }}=5 \times 10^{38} \psi\left(\frac{\eta_{e}^{\mathrm{SN}}}{10^{-3}}\right) \mathrm{erg} \mathrm{s}^{-1}
$$

SNRs inject a much larger fraction of their kinetic energy, $\eta_{p}^{\mathrm{SN}} \sim 10 \%$, into CR protons. While protons themselves do not efficiently produce nonthermal radio emissions, they collide with gases in the interstellar medium and produce pions, which promptly decay into CR electrons, among other particles. The fraction of proton power transferred to pions in the galaxy, $f_{p p}$, is determined by the properties of galaxies. In the Milky Way, $f_{p p}$ is approximately 3\%, while it can reach $\sim 10-100 \%$ 
in starburst galaxies. The total power produced by SNRs in the form of these secondary electrons is:

$$
Q_{e}^{\mathrm{SN}, \mathrm{sec} .}=8 \times 10^{37} \psi\left(\frac{f_{p p}}{10^{-2}}\right)\left(\frac{\eta_{p}^{\mathrm{SN}}}{0.1}\right) \mathrm{erg} \mathrm{s}^{-1} .
$$

In the following, we assume $f_{p p} \propto \psi^{\beta_{p p}}$ with $\beta_{p p}=0.18$ as is suggested by $\gamma$-ray data, noting that this choice does not alter out conclusions.

Young pulsars, which are produced at the time of core-collapse supernovae, are also known as efficient accelerators of $\mathrm{CR}$ electrons. Newborn pulsars have a rotational energy on the order of $10^{48}\left(P_{i} / 150 \mathrm{~ms}\right)^{-2}$, where $P_{i}$ is the initial rotation period. Over the lifetime, pulsars spin down, converting a significant fraction of their spindown power $\left(\eta_{e}^{\mathrm{PSR}} \sim 10-100 \%\right)$ to CR electrons, producing power of

$$
Q_{e}^{\mathrm{PSR}}=5 \times 10^{37} \psi\left(\frac{P_{i}}{150 \mathrm{~ms}}\right)^{-2}\left(\frac{\eta_{e}^{\mathrm{PSR}}}{0.1}\right) \mathrm{erg} \mathrm{s}^{-1}
$$

Note that the comparison between SNR and pulsar energetics is energy-dependent. Since observations of PWNe typically show a flat radio spectrum, nonthermal electrons should have a hard spectrum. In comparison, SNRs have a soft electron spectrum. Because our work focuses on the radio band, SNR contributions are likely to be dominant over young pulsars.

Unlike SNRs and young pulsars, the number of MSPs in the specific galaxy is not traced by the prompt SFR, but is more likely linked to the stellar mass. Many details of the production of CR electrons by MSPs remain unknown. Here, we estimate their energetics based on $\gamma$-ray observations. The total $\gamma$-ray luminosity of all MSPs in the Milky Way is estimated to be $\sim 10^{38} \mathrm{erg} \mathrm{s}^{-1}$ (e.g.,[7]). Denoting the energy fraction of MSPs' spindown power that goes to $\gamma$-rays and CR electrons as $\eta_{\gamma}^{\mathrm{MSP}}$ and $\eta_{e}^{\mathrm{MSP}}$ respectively, we arrive at a steady-state electron power of $\sim 10^{38}\left(\eta_{e}^{\mathrm{MSP}} / \eta_{\gamma}^{\mathrm{MSP}}\right) \mathrm{erg} \mathrm{s}^{-1}$ in the Milky Way. Assuming that the total power from MSPs in a specific galaxy is proportional to the stellar mass, we obtain

$$
Q_{e}^{\mathrm{MSP}}=2 \times 10^{37} M_{*}\left(\frac{\eta_{e}^{\mathrm{MSP}}}{\eta_{\gamma}^{\mathrm{MSP}}}\right) \mathrm{erg} \mathrm{s}^{-1},
$$

where $M_{*}$ is the stellar mass in the unit of $10^{10} M_{\odot}$ and we used the Milky Way's value of $M_{*}=5$ [8]. This estimate suggests that, while contributions from SNRs are dominant for many cases, MSPs can become important for low-SFR $(\psi \lesssim 0.05)$ and high-mass $\left(M_{*} \gtrsim 1\right)$ galaxies. We again note that this comparison depends on the spectrum of CR electrons injected by MSPs, which is largely unknown (see Sec. 4 and 5).

Having established quantitative models for the total electron power from each source class, we discuss the production of synchrotron radiation from these populations. This requires the treatment of particle cooling and escape, which should depend on various galactic properties, e.g., magnetic field, radiation, confinement time. In this work, for simplicity, we assume that the conversion efficiency of the injected electron power to the synchrotron radiation, $f_{\mathrm{syn}}$, is related to the stellar mass:

$$
f_{\mathrm{syn}}=\alpha_{\mathrm{syn}}\left(\frac{M_{*}}{10^{10} M_{\odot}}\right)^{\beta_{\mathrm{syn}}}
$$


This is motivated by a naive expectation that $f_{\text {syn }}$ is likely higher for more massive galaxies that can confine CRs for longer times.

Combining the above discussion, we can construct models of the radio luminosity at $150 \mathrm{MHz}$ (denoted as $L$, in the unit of $10^{23} \mathrm{~W} \mathrm{~Hz}^{-1}$ ). In this talk, we focus on two cases. The first model has terms that only account for the contributions from SNRs:

$$
L_{\text {model }}=\left(a_{1} \psi+a_{2} \psi^{1.18}\right) M_{*}^{\beta_{\mathrm{syn}}} .
$$

In the second model, we add the contribution from MSPs:

$$
L_{\text {model }}=\left(a_{1} \psi+a_{2} \psi^{1.18}+a_{3} M_{*}\right) M_{*}^{\beta_{\mathrm{syn}}} .
$$

We compare our models with the LOFAR data obtained by G18.

\section{Data Analysis}

We use the flux densities, SFRs, and stellar masses of 15088 SFGs analyzed by G18. Removing AGN by cross-correlating with radio-loud AGN catalog and by utilizing a modified BPT-diagram, G18 obtained 3907 galaxies that are classified as "SFGs", which we use in the following. They obtained galactic properties by fitting multi-wavelength data with MAGPHYS [9]. Detailed information is presented in G18, and the effects of systematic uncertainties are discussed also in our paper [6].

We add intrinsic source-to-source scatter to the model by assuming that the probability of finding a specific galaxy with radio luminosity $L_{i}$ is given by

$$
P_{i}\left(L_{i}\right)=\frac{1}{\left(2 \pi \sigma_{i}^{2}\right)^{1 / 2}} \exp \left(-\frac{\left|L_{i}-L_{i}^{\text {model }}\right|^{2}}{2 \sigma_{i}^{2}}\right),
$$

where $\sigma_{i}$ is defined by $\sigma_{i}^{2}=\left(c L_{i}^{\text {model }}\right)^{2}+L_{i \text {,err }}^{2}$. Here, $L_{i \text {,err }}$ is the $1 \sigma$ measurement error and $c$ is a free parameter to account for the intrinsic model dispersion. We obtain best-fit values of $a_{1}, a_{2}, a_{3}$, $\beta_{\text {syn }}$, and $c$ by minimizing the negative of the $\log$-likelihood, $-\sum_{i} \log P_{i}$, where the summention is taken for all sample SFGs. We use IMINUIT for optimization [10]. We have tested various alternative analysis in the paper, but our conclusions remain unchanged.

\section{Results}

In Fig. 1, we show the $150 \mathrm{MHz}$ luminosity distribution of SFGs, split into four mass bins. Orange (circle) points show the observed luminosities and $1 \sigma$ error bars. Light blue (square) points show the model that only includes SNR contribution. SNR-only model fails to explain sources that have bright radio luminosities but have small SFRs. Black (star) points show the predictions when MSPs are included. This model significantly improves the fit, explaining the plateau-like feature at low-SFR and high-mass sources. Quantitatively, the fit is improved by $\Delta \log (L)=524.6$.

In Table 1, we list best-fit parameters for both models. We obtain an unphysically small value of $a_{2}$ for the SNR-only model. This can be understood, because if we the data with an empirical scaling, $L \propto \psi^{\beta}$, we obtain a best-fit value of $\beta=0.976$. Among the two terms that scale as $\psi$ 

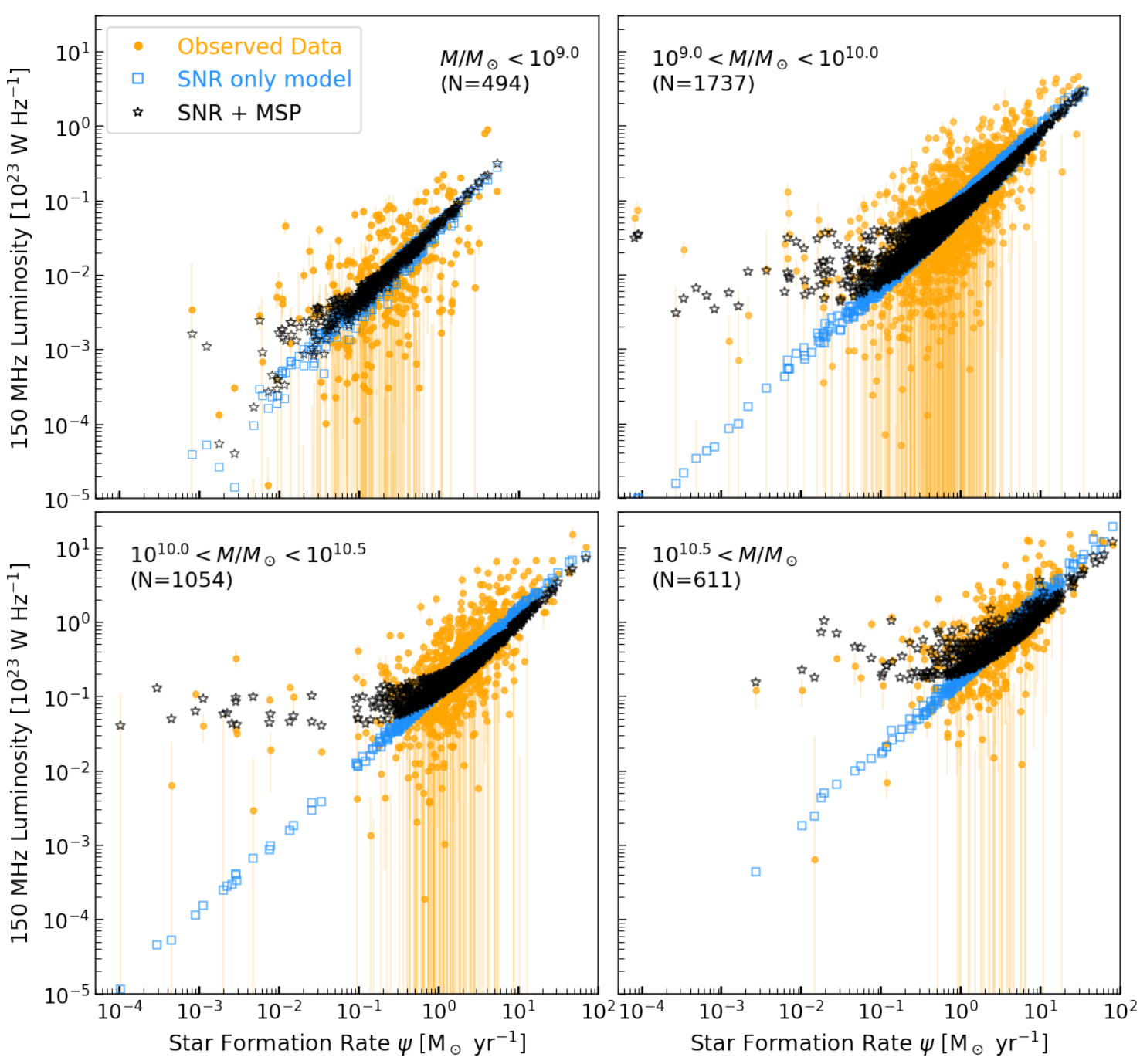

Figure 1: The $150 \mathrm{MHz}$ luminosity distribution of SFGs as a function of SFR (this and $M_{*}$ for each source are derived with MAGPHYs). The sample is split into four mass bins as labeled. Orange (circle) points show the observed LOFAR $150 \mathrm{MHz}$ luminosities and $1 \sigma$ error bars. Light blue (square) points show the model that only includes SNR contribution, which cannot fit the low-SFR data. Black (star) points show the predictions when MSPs are included, which significantly improves the fit to the LOFAR data.

and $\psi^{1.18}$, the best-fit model would only require the first term. Interestingly, the SNR+MSP model predicts a value of $a_{2}$ that is physically reasonable.

The parameter $a_{3}$ can be translated into $\eta_{e}^{\mathrm{MSP}}$ by assuming the efficiency of synchrotron emission and the spectrum of $\mathrm{CR}$ electrons. We calculate the former by assuming that massive galaxies in question are calorimetric to CR electrons. We further adopt typical Milky Way parameters to estimate the energy loss timescales. Regarding the electron spectrum, we assume an $E_{e}^{-2.0}$ spectrum up to a few $\mathrm{GeV}$, and $E_{e}^{-2.4}$ above that, which is consistent with observed radio spectral index of galaxies (e.g., [11]). Under these assumptions, we obtain

$$
\eta_{e}^{\mathrm{MSP}} \simeq 1
$$


Table 1: Best-fit parameters for our models.

\begin{tabular}{c|c|c|c|c|c}
\hline & $a_{1}$ & $a_{2}$ & $a_{3}$ & $\beta_{\text {syn }}$ & $c$ \\
\hline SNR only & 0.110 & $2.00 \mathrm{e}-10$ & - & 0.285 & 1.46 \\
SNR+MSP & 0.035 & 0.031 & 0.036 & 0.106 & 1.39
\end{tabular}

Thus, while the best-fit normalization we find does not violate the total energetics from MSPs, it appears to be rather stretching. However, there are a number of uncertainties in our modeling that may significantly affect this result. Most importantly, the energetics of galactic MSPs are highly uncertain. We have scaled the total MSPs power to the mass, based on Milky Way observations. If we instead normalized our results to M31, which has properties more consistent with quiescent galaxies (a larger stellar mass and a smaller SFR), the necessary $\eta_{e}^{\mathrm{MSP}}$ could decrease by up to a factor of about 4 , i.e., to $\simeq 30 \%$ level. Furthermore, we have ignored all the details about the evolution of the MSP populations in individual galaxies, which could induce large uncertainties and scatters. In addition, the $\mathrm{CR}$ electron spectrum could be softer than $E_{e}^{-2.0}$. In this case, the required $\eta_{e}^{\mathrm{MSP}}$ could be reduced, although it then would depend on the minimum electron energy.

\section{Discussion and Conclusions}

We propose that MSPs can be an important source of CR electrons in low-SFR and high-mass galaxies, dominating the low-end tail of the galactic radio-SFR correlation. We show that our MSP-based models significantly improve the fit to the LOFAR data, explaining the mysterious radio excess.

Our results suggest that MSPs convert a significant fraction of their spindown power to CR electrons. This idea has been supported by very recent observations. Ref. [12] finds that $\gamma$-ray emission from globular clusters exhibit a component that can be attributed to the inverse-Compton scattering of starlight via CR electrons produced by MSPs. Ref. [13] identifies emission of a similar origin in the TeV range by stacking the data of field MSPs. Additionally, Ref. [14] points out that CR electrons from MSPs could explain radio and $\gamma$-ray observations of the Galactic Center.

Our results have broad implications:

Radio-SFR Correlation: The tightness of the radio-SFR correlation has raised an expectation that the radio luminosity can be a useful SFR tracer. Our results suggest that this correlation may break down for low-SFR and high-mass galaxies, limiting the applicability. At the same time, we suggest that the study of radio-SFR correlation may be used to investigate the population of MSPs in galaxies and their CR production.

Radio-far-infrared (FIR) Correlation: If MSPs contribute radio luminosities, one might expect that the radio-FIR correlation should also break down at low FIR luminosity. However, this is not clearly seen in the LOFAR data in G18 (although uncertainties in the FIR luminosities are large). This is naturally expected. For massive galaxies, in which the radio luminosity is enhanced by MSPs, the FIR luminosity is substantially enhanced due to the heating of the dust by old stellar populations $[15,16]$. Therefore, even with low-SFR, massive galaxies can be bright both in radio and FIR. 
Radio and $\gamma$-ray emission from M31: Although G18 has removed AGN based on BPT-diagram diagnostics, potential contributions from AGN remain a concern. One promising way to separate AGN emission is to use nearby, spatially resolved galaxies. In particular, M31 serves as a good target. Ref. [17] points out that the radio and $\gamma$-ray observations of M31 bulge suggest that it hosts an CR electron power of $\sim 10^{39} \mathrm{erg} \mathrm{s}^{-1}$, while SNRs can only inject $\sim 5 \times 10^{37} \mathrm{erg} \mathrm{s}^{-1}$. Interestingly, our model predicts that MSPs can inject $\sim 8 \times 10^{38} \mathrm{erg} \mathrm{s}^{-1}$. Therefore, radio and $\gamma$-ray emission from the M31 bulge could be explained by the contributions from CR electrons produced by MSPs.

Local CR electron and positron measurements: If MSPs efficiently produce CR electrons, they may contribute to the local $e^{ \pm}$flux. In particular, MSPs could significantly contribute to the positron excess, as pointed out in Ref. [18].

\section{References}

[1] Condon, J. J. 1992, ARAA, 30, 575. doi:10.1146/annurev.aa.30.090192.003043

[2] Gürkan, G., Hardcastle, M. J., Smith, D. J. B., et al. 2018, Monthly Notices of Royal Astronomical Society, 475, 3010. doi:10.1093/mnras/sty016

[3] Tauris, T. M. \& van den Heuvel, E. P. J. 2006, Compact stellar X-ray sources, 623

[4] Lorimer, D. R. 2013, Neutron Stars and Pulsars: Challenges and Opportunities after 80 years, 291, 237. doi:10.1017/S1743921312023769

[5] Fragos, T., Lehmer, B., Tremmel, M., et al. 2013, The Astrophysical Journal, 764, 41. doi:10.1088/0004-637X/764/1/41

[6] Sudoh, T., Linden, T., \& Beacom, J. F. 2021, Physical Review D, 103, 083017. doi:10.1103/PhysRevD.103.083017

[7] Eckner, C., Hou, X., Serpico, P. D., et al. 2018, The Astrophysical Journal, 862, 79. doi:10.3847/1538-4357/aac029

[8] Licquia, T. C. \& Newman, J. A. 2015, The Astrophysical Journal, 806, 96. doi:10.1088/0004$637 \mathrm{X} / 806 / 1 / 96$

[9] da Cunha, E., Charlot, S., \& Elbaz, D. 2008, Monthly Notices of Royal Astronomical Society, 388, 1595. doi:10.1111/j.1365-2966.2008.13535.x

[10] James, F. \& Roos, M., Computer Physics Communications 10, 343 (1975).

[11] Chyży, K. T., Jurusik, W., Piotrowska, J., et al. 2018, Astronomy \& Astrophysics, 619, A36. doi:10.1051/0004-6361/201833133

[12] Song, D., Macias, O., Horiuchi, S., et al. 2021, arXiv:2102.00061

[13] Hooper, D. \& Linden, T. 2021, arXiv:2104.00014

[14] Gautam, A., Crocker, R. M., Ferrario, L., et al. 2021, arXiv:2106.00222 
[15] Salim, S., Dickinson, M., Michael Rich, R., et al. 2009, The Astrophysical Journal, 700, 161. doi:10.1088/0004-637X/700/1/161

[16] Calzetti, D., Wu, S.-Y., Hong, S., et al. 2010, The Astrophysical Journal, 714, 1256. doi:10.1088/0004-637X/714/2/1256

[17] McDaniel, A., Jeltema, T., \& Profumo, S. 2019, Physical Review D, 100, 023014. doi:10.1103/PhysRevD.100.023014

[18] Kisaka, S. \& Kawanaka, N. 2012, Monthly Notices of Royal Astronomical Society, 421, 3543. doi:10.1111/j.1365-2966.2012.20576.x 